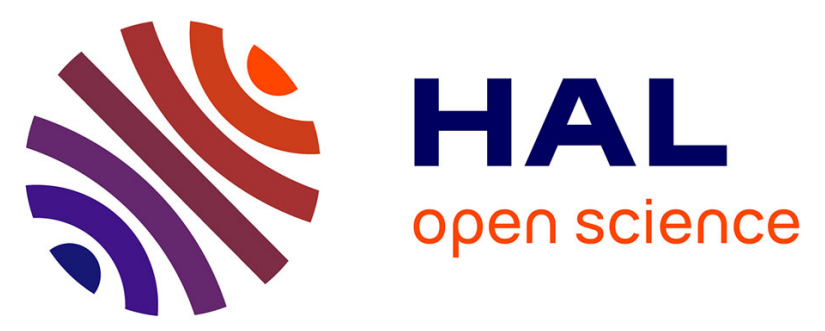

\title{
Observation of magneto-optical second-harmonic generation with surface plasmon excitation in ultrathin $\mathrm{Au} / \mathrm{Co} / \mathrm{Au}$ films \\ V. V. Pavlov, Gilles Tessier, C. Malouin, Patrick Georges, Alain Brun, D. Renard, P. Meyer, J. Ferre, Pierre Beauvillain
}

\section{To cite this version:}

V. V. Pavlov, Gilles Tessier, C. Malouin, Patrick Georges, Alain Brun, et al.. Observation of magnetooptical second-harmonic generation with surface plasmon excitation in ultrathin $\mathrm{Au} / \mathrm{Co} / \mathrm{Au}$ films. Applied Physics Letters, 1999, 75 (2), pp.190-192. 10.1063/1.124315 . hal-00761541

HAL Id: hal-00761541

https://hal-iogs.archives-ouvertes.fr/hal-00761541

Submitted on 15 Apr 2016

HAL is a multi-disciplinary open access archive for the deposit and dissemination of scientific research documents, whether they are published or not. The documents may come from teaching and research institutions in France or abroad, or from public or private research centers.
L'archive ouverte pluridisciplinaire HAL, est destinée au dépôt et à la diffusion de documents scientifiques de niveau recherche, publiés ou non, émanant des établissements d'enseignement et de recherche français ou étrangers, des laboratoires publics ou privés. 


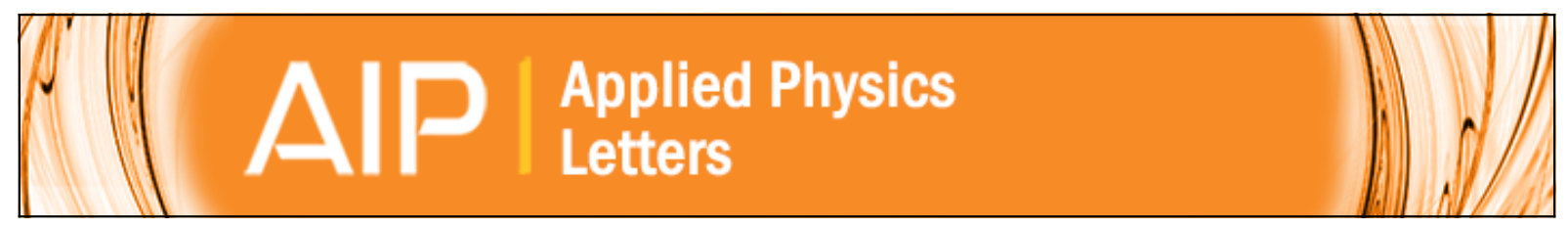

Observation of magneto-optical second-harmonic generation with surface plasmon excitation in ultrathin Au/Co/Au films

V. V. Pavlov, G. Tessier, C. Malouin, P. Georges, A. Brun, D. Renard, P. Meyer, J. Ferré, and P. Beauvillain

Citation: Applied Physics Letters 75, 190 (1999); doi: 10.1063/1.124315

View online: http://dx.doi.org/10.1063/1.124315

View Table of Contents: http://scitation.aip.org/content/aip/journal/apl/75/2?ver=pdfcov

Published by the AIP Publishing

\section{Articles you may be interested in}

Magneto-optic surface plasmon resonance of $\mathrm{Au} / \mathrm{lrMn} / \mathrm{Co} / \mathrm{Au}$ exchange biased layer systems

J. Appl. Phys. 116, 064502 (2014); 10.1063/1.4892563

Enhancement of the magnetic modulation of surface plasmon polaritons in $\mathrm{Au} / \mathrm{Co} / \mathrm{Au}$ films

Appl. Phys. Lett. 97, 183114 (2010); 10.1063/1.3512874

Surface plasmon resonance and magneto-optical enhancement on Au-Co nanocomposite thin films

J. Appl. Phys. 107, 103924 (2010); 10.1063/1.3428470

Near-field second-harmonic imaging of thin ferromagnetic films

Appl. Phys. Lett. 85, 6341 (2004); 10.1063/1.1842369

One monolayer-periodic oscillations in the magnetization induced second harmonic generation signals during the growth of Co films on $\mathrm{Cu}(001)$

J. Appl. Phys. 85, 5288 (1999); 10.1063/1.369856

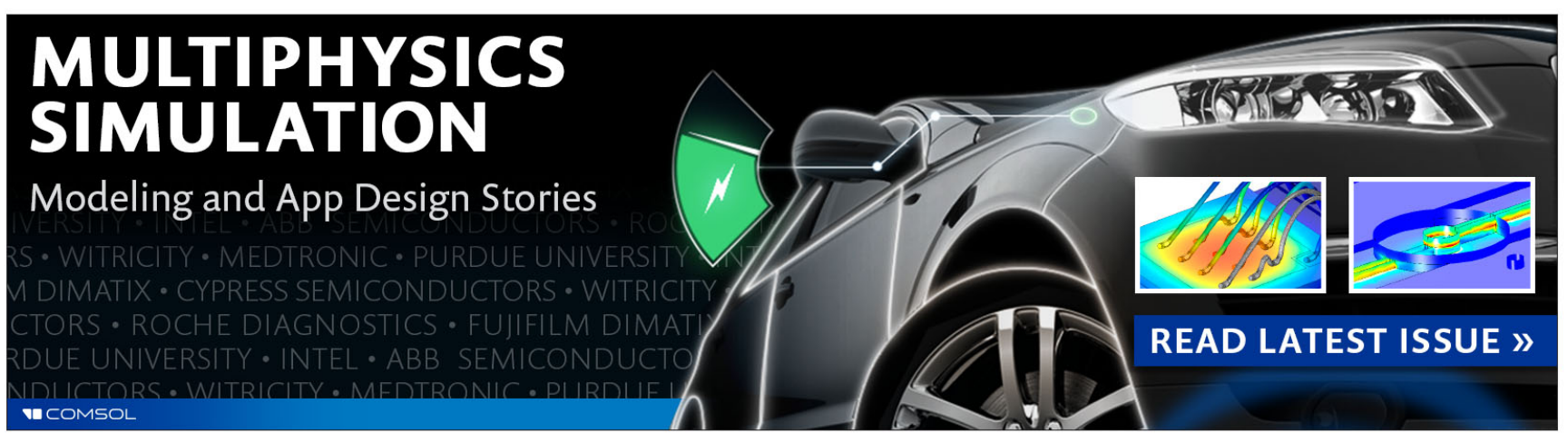




\title{
Observation of magneto-optical second-harmonic generation with surface plasmon excitation in ultrathin $\mathrm{Au} / \mathrm{Co} / \mathrm{Au}$ films
}

\author{
V. V. Pavlov \\ A. F. Ioffe Physical Technical Institute of the Russian Academy of Sciences, 194021 St. Petersburg, Russia \\ G. Tessier, a) C. Malouin, P. Georges, A. Brun, and D. Renard \\ Laboratoire Charles Fabry de l'Institut d'Optique, UMR CNRS 8501, BP 147, 91403 Orsay Cédex, France \\ P. Meyer and J. Ferré \\ Laboratoire de Physique des Solides, UMR CNRS 8502, Université de Paris-Sud, 91405 Orsay Cédex, \\ France \\ P. Beauvillain \\ Institut d'Electronique Fondamentale, UMR CNRS 8622, Université de Paris-Sud, 91405 Orsay Cédex, \\ France
}

(Received 11 February 1999; accepted for publication 17 May 1999)

\begin{abstract}
Magnetization-induced second-harmonic generation with surface plasmon excitation in an ultrathin $\mathrm{Au} / \mathrm{Co} / \mathrm{Au}$ multilayer structure has been investigated. The resonant coupling of surface plasmons with the fundamental light results in drastic changes of the second-harmonic intensity and a sign reversal of nonlinear magneto-optical effects. Model analysis of the observed phenomena is given on the basis of the multiple interference of interface nonlinear contributions calculated using the Green's functions formalism. (C) 1999 American Institute of Physics. [S0003-6951(99)01328-5]
\end{abstract}

Surface collective electron oscillations, also known as surface plasmons (SP), can be excited in noble metals below the plasma frequency and may give rise to a variety of linear and nonlinear phenomena. ${ }^{1}$ The coupling of the electric field at optical frequencies with SP in metallic multilayer films results in an increase of the linear magneto-optical effects. ${ }^{2}$ It has been shown experimentally and theoretically that the optical second-harmonic generation (SHG) is strongly enhanced due to the SP excitation. ${ }^{1,3}$ Since the observation of magnetization-induced $\mathrm{SHG}^{4}$ it has been established that SHG is a powerful method to study magnetic surfaces and interfaces. This method was intensively used for investigations of new nonlinear magneto-optical phenomena in different film compositions, ${ }^{5,6}$ showing that magnetization-induced SHG is very sensitive to the crystallographic, magnetic, and electronic structure of thin films.

In this letter we present the experimental observation of nonlinear magneto-optical phenomena related to the surface plasmon excitation in an ultrathin $\mathrm{Au} / \mathrm{Co} / \mathrm{Au}$ multilayer structure. The measurements have been done using the attenuated total reflection technique (ATR) in the Kretschmann geometry. ${ }^{1}$ The coupling of the SP with light at the fundamental frequency gives rise to drastic changes of secondharmonic $(\mathrm{SH})$ intensity. $\mathrm{SH}$ magnetic contrast exhibits a sign reversal when the SP are excited. We developed a model on the basis of the Green's functions (GF) approach, which allows us to describe different contributions of the film interfaces to the SHG. Model calculations were found to be in good agreement with the observed phenomena.

In crystallographically centrosymmetric layers the SHG is only allowed, in the electric-dipole approximation, at surfaces and interfaces where the space-inversion symmetry is

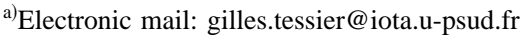

broken. In the presence of a magnetization $\mathbf{M}$, the nonlinear polarization $\mathbf{P}^{2 \omega}$ can be written as

$$
P_{i}^{2 \omega}=\epsilon_{0} \chi_{i j k}^{N} E_{j} E_{k} \pm \epsilon_{0} \chi_{i j k}^{M}( \pm \mathbf{M}) E_{j} E_{k}
$$

where $E_{j}$ and $E_{k}$ are components of the optical electric field at the fundamental frequency $\omega$. The nonlinear susceptibility tensors $\chi_{i j k}^{N}$ and $\chi_{i j k}^{M}$ describe nonmagnetic and magnetic contributions to the nonlinear polarization $\mathbf{P}^{2 \omega}$, respectively. Neglecting dissipation in the medium, $\chi_{i j k}^{N}$ is a real tensor element and $\chi_{i j k}^{M}$ is a pure imaginary. ${ }^{7}$ In the presence of absorption, both tensors are complex, thus allowing an interference between the two contributions. This interference gives rise to new nonlinear phenomena such as nonlinear magneto-optical rotation ${ }^{5}$ and nonlinear circular dichroism.

The ultrathin $\mathrm{Au} / \mathrm{Co} / \mathrm{Au}$ films were deposited on a 1-mm-thick float glass substrate in a high vacuum chamber, following the procedure described in Ref. 8. In the $\mathrm{Au}(3$ $\mathrm{nm}) / \mathrm{Co}(3 \mathrm{~nm}) / \mathrm{Au}(25 \mathrm{~nm}) / \mathrm{glass}$ structure, the easy magnetization axis was located in the film plane. Pulsed coils producing an in-plane magnetic field up to $1 \mathrm{kOe}$ were specially designed to magnetize this sample at saturation. The glass substrate was optically coupled to a half-cylindrical glass lens using a refractive index adaptation liquid.

The experimental setup and the geometry of measurements in the ATR configuration are shown in Fig. 1. The mode-locked Ti:sapphire laser pumped by an Ar laser was used as a source of 100 fs light pulses with a repetition frequency of $86 \mathrm{MHz}$ at the wavelength of $\lambda=800 \mathrm{~nm}$. The beam with an average power of $30 \mathrm{~mW}$ was focused on the sample in a spot of about $100 \mu \mathrm{m}$ in diameter. In front of the sample a RG715 filter was used in order to eliminate a possible parasitic SHG generated at the surfaces of the optical 


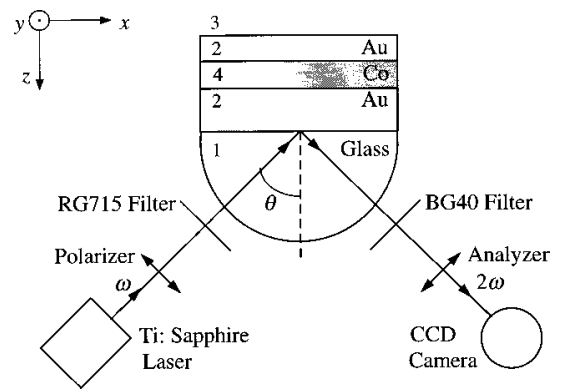

FIG. 1. Schematics of the experimental setup.

elements. After the sample, a BG40 filter was used to block the fundamental light. The SH intensity generated by the $\mathrm{Au} / \mathrm{Co} / \mathrm{Au}$ film was measured using a high-sensitivity cooled charge-coupled device camera. We used $p$-polarized fundamental light (TM mode), which excites SP near an incidence angle $\theta_{p}$ with a wave vector $k_{p}$ defined by the SP dispersion equation ${ }^{1}$

$$
k_{p}=\frac{\omega}{c} \sqrt{\frac{\epsilon_{1} \epsilon_{2}}{\epsilon_{1}+\epsilon_{2}}},
$$

where $\epsilon_{1}$ and $\epsilon_{2}$ are the dielectric constants of the two media at the interface where SP are excited. SP may be coupled with evanescent optical waves from a half-cylindrical glass lens at the angle

$$
\theta_{p}=\arcsin \left(\frac{k_{p} c}{\omega \sqrt{\epsilon}}\right)
$$

where $\epsilon$ is the dielectric constant of the glass. For interfaces between optically isotropic media with $p$-polarized excitation, when there are only $x$ and $z$ components in the fundamental electric field (see Fig. 1), the nonlinear polarization $\mathbf{P}^{2 \omega}$ can be described for the longitudinal geometry $(\mathbf{M} \| x)$ by the matrix expression

$$
\mathbf{P}^{2 \omega}=\epsilon_{0}\left(\begin{array}{ccc}
0 & \chi_{x x z}^{N} & 0 \\
\chi_{y x x}^{M} & 0 & \chi_{y z z}^{M} \\
\chi_{z x x}^{N} & 0 & \chi_{z z z}^{N}
\end{array}\right)\left(\begin{array}{c}
E_{x} E_{x} \\
2 E_{x} E_{z} \\
E_{z} E_{z}
\end{array}\right) .
$$

For the transversal geometry, when $\mathbf{M} \| y, \mathbf{P}^{2 \omega}$ can be written as

$$
\mathbf{P}^{2 \omega}=\epsilon_{0}\left(\begin{array}{ccc}
\chi_{x x x}^{M} & \chi_{x x z}^{N} & \chi_{x z z}^{M} \\
0 & 0 & 0 \\
\chi_{z x x}^{N} & \chi_{z x z}^{M} & \chi_{z z z}^{N}
\end{array}\right)\left(\begin{array}{c}
E_{x} E_{x} \\
2 E_{x} E_{z} \\
E_{z} E_{z}
\end{array}\right) .
$$

The nonzero tensor components are determined on the basis of symmetry considerations. One must note that, due to the symmetry, $\chi_{y z z}^{M}=-\chi_{x z z}^{M}$ in Eqs. (4) and (5).

The variations of the reflectivity of the $\mathrm{Au} / \mathrm{Co} / \mathrm{Au}$ film at the fundamental light frequency versus the angle of incidence $\theta$ are shown in Fig. 2(a). The signal increases up to the total internal reflection angle $\theta_{t} \simeq 43.5^{\circ}$ and then strongly decreases down to the angle $\theta_{p} \simeq 45^{\circ}$. The sharp minimum in the intensity of the reflected light indicates that SP are excited near this angle. A similar behavior of the reflected light at $\lambda=632.8 \mathrm{~nm}$ in $\mathrm{Au} / \mathrm{Co} / \mathrm{Au}$ film has already been observed by Safarov et $\mathrm{al}^{2}$. A theoretical modelization of the reflection coefficients and magneto-optical effects in $\mathrm{Au} / \mathrm{Co} / \mathrm{Au}$

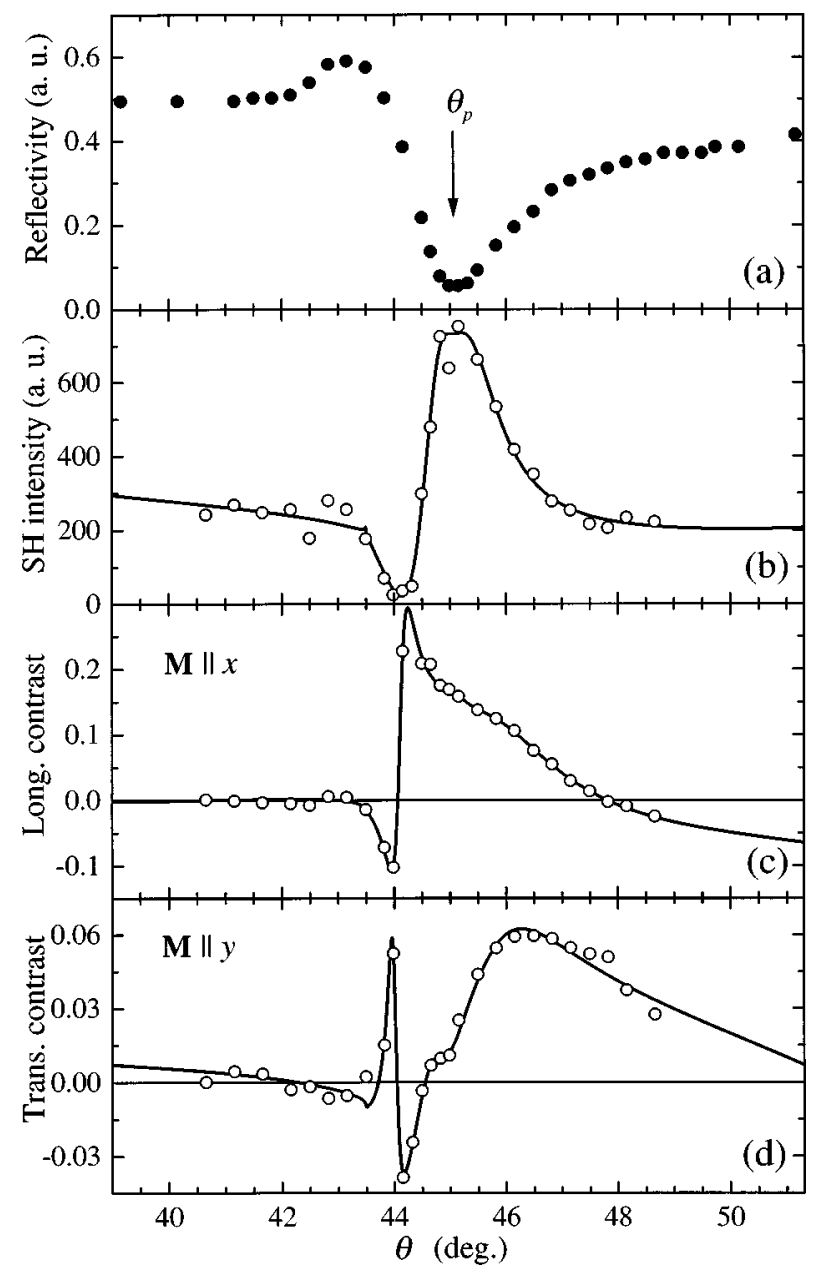

FIG. 2. Variations with the incidence angle $\theta$ of the reflectivity at the fundamental frequency (a), the nonmagnetic SHG intensity (b), and the SH magnetic contrast in longitudinal (c) and transversal (d) geometries.

films in the ATR configuration on the basis of the GF technique has been achieved by Kosobukin. ${ }^{9}$ It was established that in the $\mathrm{Au} / \mathrm{Co} / \mathrm{Au} / \mathrm{glass}$ structure $\mathrm{SP}$ are excited at the air/Au interface. The coupling of the light with SP gives rise to a strong enhancement of the magneto-optical linear Kerr effect. $^{2}$

The total SH intensity measured in longitudinal geometry $(\mathbf{M} \| x)$ with $p$-polarized input and $p$-polarized output is shown by dots in Fig. 2(b). According to Eq. (4) there is no magnetic contribution to the nonlinear polarization in this configuration. The $\mathrm{SH}$ signal has a strong minimum at the angle $\theta \simeq 44^{\circ}$. It reaches a maximum near $\theta_{p}$, decreases, and is constant for angles $\theta>47^{\circ}$.

The dots in Fig. 2(c) show the experimental longitudinal (M\|) SH magnetic contrast $\rho$, defined as

$$
\rho=\frac{I^{2 \omega}(+\mathbf{M})-I^{2 \omega}(-\mathbf{M})}{I^{2 \omega}(+\mathbf{M})+I^{2 \omega}(-\mathbf{M})}=\frac{2\left|\chi^{N}\right|\left|\chi^{M}\right| \cos \varphi}{\left|\chi^{N}\right|^{2}+\left|\chi^{M}\right|^{2}},
$$

versus the angle $\theta$ ( $\varphi$ is the optical phase difference between the even and odd tensor elements). We chose the position of the analyzer $\alpha=80^{\circ}$ in order to obtain a good signal-to-noise ratio ( $\alpha=0$ corresponds to the $p$ polarization). The contrast has a sharp jump at $\theta \simeq 44^{\circ}$. Near the angle of SP excitation $\theta_{p}, \rho$ decreases drastically and then changes sign at $\theta$ $\simeq 48^{\circ}$. The same type of measurements were done in trans- 
versal configuration ( $\mathbf{M} \| y)$ for $p$-input and $p$-output polarizations and are shown in Fig. 2(d). The sign of the transversal SH contrast changes three times in a narrow angle interval $\theta_{t}<\theta<45^{\circ}$. Then, $\rho$ increases strongly and has a broad maximum at $\theta \simeq 46.5^{\circ}$.

In order to explain our results, we developed a model involving multiple interference of the different interface contributions to SHG, which can be calculated using the GF technique. The GF approach was used for general description of SHG from surfaces and interfaces by Guyot-Sionnest, Chen, and Shen ${ }^{10}$ and for analysis of the SHG dispersion at simple metal surfaces by Liebsch and Schaich. ${ }^{11}$ The GF formalism is a convenient way for the consideration of the light propagation problem in multilayer structures, when the layer thickness is much less than the light wavelength. In our model we took into account the coupling of SP with the fundamental light, using a theory developed for the SP excitation in trilayer metallic systems. ${ }^{9}$ The electric field $E_{i}^{2 \omega}$ of the outcoming SH wave in the glass cylindrical lens can be represented as a sum of contributions from the interfaces of the film:

$$
\begin{aligned}
E_{i}^{2 \omega} \propto & T^{24} T^{42} T^{21} \chi_{i j k}^{32} F_{j}^{32} F_{k}^{32} e^{2 i\left(K_{2} d_{2}^{\prime}+K_{4} d_{4}+K_{2} d_{2}\right)} \\
& +T^{42} T^{21} \chi_{i j k}^{24} F_{j}^{24} F_{k}^{24} e^{2 i\left(K_{4} d_{4}+K_{2} d_{2}\right)} \\
& -T^{21} \chi_{i j k}^{24} F_{j}^{42} F_{k}^{42} e^{2 i K_{2} d_{2}+} \chi_{i j k}^{21} F_{j}^{21} F_{k}^{21},
\end{aligned}
$$

where $T^{a b}$ are transmission coefficients for the SH light at the $a b$ interface, and $F_{j}^{a b}$ and $F_{k}^{a b}$ are continuous local integral functions of the electric fields at the fundamental frequency. The numbering of interfaces is given in Fig. 1: 32 is the air/Au, 24 is the Au/Co interface, etc. $K_{2}$ and $K_{4}$ are the $z$ components of the SH wave vectors in Au and Co. $d_{2}, d_{2}^{\prime}$, and $d_{4}$ are the thicknesses of the Au buffer and cover layers and of the Co layer, respectively. One can suppose that $\chi_{i j k}^{42}=-\chi_{i j k}^{24}$, because the $\mathrm{Au} / \mathrm{Co}$ and the $\mathrm{Co} / \mathrm{Au}$ interfaces are perfectly symmetrical. The quantities $T^{a b}$ and $F_{i}^{a b}$ were calculated using, respectively, Eqs. (A7) and (3.16) in Ref. 9. The complex refractive indices of $\mathrm{Au}$ and $\mathrm{Co}$ were taken from Ref. 12. The SH intensity was calculated as $I^{2 \omega}$ $\propto\left|E_{x}^{2 \omega} \cos \theta+E_{z}^{2 \omega} \sin \theta\right|^{2}$ for the $p$-polarized SH light and as $I^{2 \omega} \propto\left|E_{y}^{2 \omega}\right|^{2}$ for the $s$ polarization. We developed a program for the fitting procedure of the experimental data. Unknown components of the complex nonlinear tensors $\chi_{i j k}^{a b}$ were used as fit parameters. Nonmagnetic components of $\chi_{i j k}^{a b}$ were found fitting the data shown in Fig. 2(b). Then, nonmagnetic components of $\chi_{i j k}^{a b}$ were fixed and the fitting procedure was continued for the SH magnetic contrast using Eq. (6). The calculated curves are shown in Figs. 2(b)-2(d) by the solid lines. One can see a good agreement of the modelization with the experiment.

We demonstrated experimentally the magnetizationinduced second-harmonic generation with the surface plasmon excitation in ultrathin $\mathrm{Au} / \mathrm{Co} / \mathrm{Au}$ structures. In the electric-dipole approximation the second-harmonic generation is allowed at magnetic and nonmagnetic interfaces in these films. At the angle $\theta_{p}$, due to the coupling of SP with the fundamental light, the fundamental electric field is strongly enhanced at the nonmagnetic air/ $\mathrm{Au}$ interface and redistributed to the magnetic $\mathrm{Au} / \mathrm{Co}$ and $\mathrm{Co} / \mathrm{Au}$ interfaces. This results in an enhancement of both the magnetizationinduced and the nonmagnetic SHG. Drastic changes in the distribution of the fundamental field at the magnetic interfaces near $\theta_{p}$ result in a sign reversal of the SH magnetic contrast in the longitudinal and transversal geometries. Model calculations based on the Green's functions technique are shown to be in good agreement with the experimental results.

The authors wish to acknowledge fundings from the $\mathrm{BQR}$ of the Universite Paris XI, the TMR network, Contract No. ERBFMRXCT98-0015 (NOMOKE), ULTIMATECH, and Contract No. INTAS 97-0705. The work of one of the authors (V.V.P.) was supported by a grant from the MÉNESR and by the RFBR. The work of one of the authors (C.M.) was supported by the MAÉ (Bourse Brézin).

${ }^{1}$ Surface Polaritons, edited by V. M. Agranovich and D. L. Mills (NorthHolland, Amsterdam, 1982).

${ }^{2}$ V. I. Safarov, V. A. Kosobukin, C. Hermann, G. Lampel, and J. Peretti, Phys. Rev. Lett. 73, 3584 (1994).

${ }^{3}$ C. K. Chen, A. R. B. de Castro, and Y. R. Shen, Opt. Lett. 4, 393 (1979), and references cited therein.

${ }^{4}$ J. Reif, J. C. Zink, C.-M. Schneider, and J. Kirschner, Phys. Rev. Lett. 67, 2878 (1991)

${ }^{5}$ Th. Rasing, J. Magn. Magn. Mater. 175, 35 (1997), and references cited therein.

${ }^{6}$ T. M. Crawford, C. T. Rogers, T. J. Silva, and Y. K. Kim, Appl. Phys. Lett. 68, 1573 (1996).

${ }^{7}$ R.-P. Pan, H. D. Wei, and Y. R. Shen, Phys. Rev. B 39, 1229 (1989).

${ }^{8}$ D. Renard and G. Nihoul, Philos. Mag. B 55, 75 (1987).

${ }^{9}$ V. A. Kosobukin, J. Magn. Magn. Mater. 153, 397 (1996)

${ }^{10}$ P. Guyot-Sionnest, W. Chen, and Y. R. Shen, Phys. Rev. B 33, 8254 (1986).

${ }^{11}$ A. Liebsch and W. L. Schaich, Phys. Rev. B 40, 5401 (1989).

${ }^{12}$ P. B. Johnson and R. W. Christy, Phys. Rev. B 6, 4370 (1972); 9, 5056 (1974). 